\title{
Harnessing the Power of Deep Learning to Save Animals
}

\author{
B. V. S. Mridula \\ Software Engineer
}

\author{
Padma Bonde \\ Member IEEE
}

\begin{abstract}
During the last decade, AI has become the most important field for the researchers to provide a solutions for real world's challenges, resulting in the new emerged field of deep learning which make the system more efficient and optimized .This paper discusses the expansion of deep learning towards the welfare of animal life considering ways to save them and monitoring their count and health conditions. Techniques like image classification and object detection can provide plethora of information that can assist in conducting study on various aspects by identifying and classifying these animal species from thousands of images captured by drones or cameras which could be very strenuous and tedious to be done by humans.
\end{abstract}

\section{General Terms}

Saving Animals, Deep learning Techniques, Pattern Recognition

\section{Keywords}

Deep Learning, Animals, Neural Networks, Image Recognition, Video Data analysis, Animal Image Detection, Animal Image Classification.

\section{INTRODUCTION}

Manual data analysis by complete human intervention possess a number of challenges, in such case deep learning could prove to be very effective in addressing these challenges and in finding innovative and efficient optimized solutions by reducing human effort. Deep learning is a methodology that induces artificially built intelligence into functions that mimic the functionality of human brain in processing data. Likewise humans improve at an activity by continuous repetitions of tasks associated with it, a deep learning model also learns by continuously iterating through training data multiple numbers of times and improving its intelligence on its own at every stage and thus the intelligence gained could be used to make decisions about other data.

Inspired by the nerve cells (neurons) that make up the human brain, neural networks comprise layers (neurons) that are connected in adjacent layers to each other resulting in deeper network with more layers. Human brain comprising of number of a large network of neurons, when a neuron receives a signal associated with any action, it passes an excitatory signal to all its connecting neurons and if the threshold of the connecting neuron reaches, it in turn fires its further connecting neurons and in this way signals are transmitted from one neuron to another in a human neural network.

In case of an artificial neural network, each neuron is associated with some weight and it exerts equivalent weight on its connecting neurons. Thus the effect exerted on a neuron is directly proportional to the weight associated with its preceding connecting neurons. An artificial neural network comprises of an input layer followed by a single or multiple hidden layer and a final output layer. Below figure (figure 1) illustrates a neural network with 3 node input layer, two 6 node hidden layers and finally a single node output layer

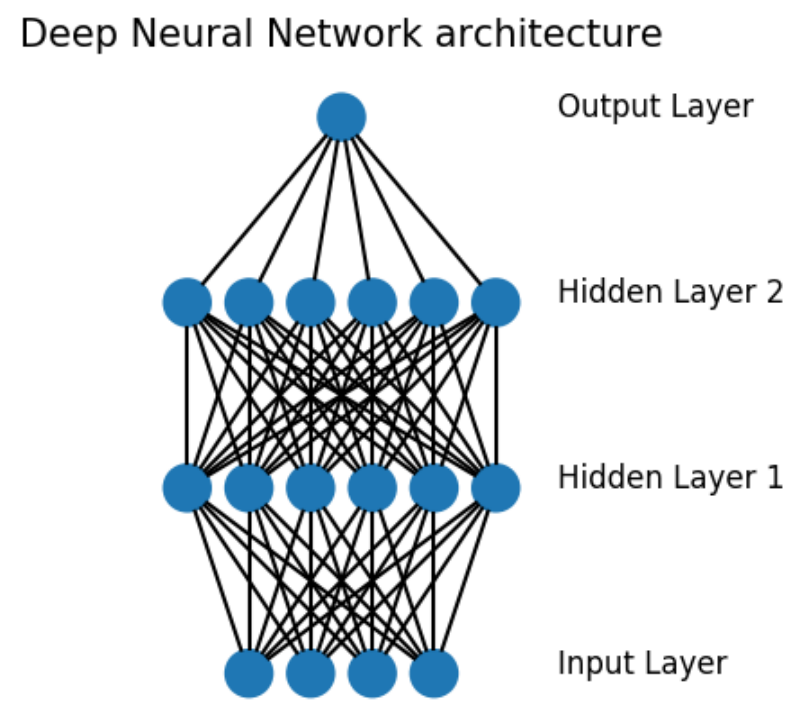

Fig 1: Deep Neural network

The training of a model begins by allocating each node in the network with some random weight, then for each input, the output is computed using forward propagation from left to right. Then, the final output from last node is compared to the actual output from training data and the error is measured for each input using loss function. Using back propagation technique from right to left, the error is propagated back to each node and the contribution of each node in the error is computed and finally the weight is adjusted using gradient decent. This process is repeated until the error is minimized or approaches to zero

\section{DEEP LEARNING TECHNIQUES}

Image classification and object detection are some of the broadly used techniques of deep learning. In a neural network comprising of multiple layers, each layer is responsible for extracting one or more features of the image. When a model is fed with a large dataset of labelled images with a large number of identifiable parameters, it will start recognizing patterns associated uniquely with each category. This is known as supervised learning, where a model learns by looking at similar patterns in one category and which are absent from other categories, as a result, when it encounters the same set of distinct features, it is able to differentiate and predict the image. Greater the number of comparable parameters and training input dataset, greater is the accuracy of the prediction of the model.

Object detection is another methodology where a model is fed with an input image; it returns the width, height of the bounding box which contains the object. If a model is trained to recognize a particular object by furnishing it with a set of images of the same object viewed from different angles, it should be able to locate the object when an image containing that object is provided as input. Further pattern recognition is also one of the 
widely used methodology in the field of deep learning, where each layer in the neural network is responsible of identifying a set of pattern of features associated with the given input which collectively make the learned pattern unique. Once the model is able to associate a certain sequence of features for a given pattern and learn the possible outcome from training data, it will be able to deduce the output when an input with similar features are provided as input.

\section{WIDELY USED APPLICATIONS OF DEEP LEARNING}

Methods like Image classification and object detection have been very well used for Industrial and commercial purposes like anomaly detection in manufacturing parts, integrating image recognition to improve their users experience by telecoms companies. They add value to their services by offering image organization and classification for photo libraries, which helps them attract and retain their customers. Other successful and widespread applications include real time theft detection and prevention using face recognition algorithms,[1] In agricultural industry, image processing is been utilizing in different ways to identify the crop, plant, leaves, flower, fruits etc. as well as to identify the disease. Digital image processing is a technique used for enhancement of the image to improve the agriculture product automatic detection.

\section{ANIMAL IDENTIFICATION AND CLASSIFICATION USING DEEP LEARNING}

The implementation of Deep learning could be expanded for animal identification and classification. However the scope has been left unexplored or minimally explored towards the welfare of animals. With the current difficulty in obtaining detailed, accurate and up to date information about the wildlife in forests and marine life in oceans and seas governing their behaviour in that area, the speed and power of image classification and object detection could be leveraged to classify and identify images of animals and categorize them on the basis of a set of distinct features. For example, the Snapshot Serengeti (hereafter, SS) project has 3.2 million images and the images have been labelled by a group of 28,000 registered and 40,000 unregistered volunteer "citizen scientists" [2]. Currently, it takes 2-3 months for these thousands of people to classify each 6-month batch of images GPU's and motion sensor cameras fit in forests or underwater are able to collect large number of images each day. If a neural network is well trained to identify distinct pattern in the images of each animal, it can not only differentiate between two species but also two different animals of same species.

\section{USE CASES WITH DEEP LEARNING 5.1 Curbing road accidents caused by stray animals}

Although it accounts for a smaller percentage, but there have been a noticeable number of cases recorded for accidents involving animals mistakenly coming in the way of drivers. Either it is the case of a wild animal accidentally crossing the highway roads in the night or any stray animal being hit by a rushing vehicle on the busy road, each of these cases possesses a potential risk for both the driver and the animal. Techniques like pattern recognition could be used where the model can be trained to identify pattern associated with certain features like number of stray animals found in a particular locality, their population growth rates, type of the animal, locality conditions against the output as number of accidents occurred in that particular locality, it would be able to predict the possible risk locations of accidents on the basis of the number of animals and the locality. If such model is developed, not only the lives of people but also these innocent animals could also be saved. Also, on identifying the areas densely populated by these stray animals, it will be easier to keep a check on the count of these animals by immediately handing them over to animal welfare associations.

5.2 Saving endangered species from poaching Drones and GPU's could be harboured to collect plenty of wildlife images and videos. Using deep learning neural network model trained with these collected images and videos can track, pinpoint and even predict the paths of animals, vehicles and poachers, learning more and more as time goes on. Also, the ability of the model to discriminate between visible and infrared light, it would be easier to know if the recording has been made during the day or at night. Once the model is able to identify a particular animal with respect to it features, it could trace down the path travelled by each animal (as the model can even differentiate between two animals of same species) and could possibly find any abnormality with the movement patterns. Also training the model with known routes followed by poachers, with this abnormality, the model could match the abnormal path with the possible poachers path to know whether the animal is being poached or not.

\subsection{Studying the behaviour of rare or elusive animal species}

Some animal species like sea cow and snow leopard are at the verge of extinction. Their low population and elusive nature makes it difficult to keep a track of them. As for example in the case of sea cows, scientists had to spend days peering out of small planes to count populations, which was expensive and sometimes hazardous. Now with the help of aerial photography, it has become easier to capture thousands of images over the oceans, but the real challenge is to identify sea cows in those photos. Dr. Amanda Hodgson of Murdoch University teamed up with Dr. Frederic Maire, a computer scientist at Queensland University of Technology and using tensorflow built a detector that could learn to find sea cows in these photos automatically [3].

Snow leopard which is also a potentially endangered animal is very difficult to study due to its remote habitat and highly elusive nature. Biologists have been using camera trap images for conducting study, but classifying images manually consumed about $300 \mathrm{hrs}$ per camera survey. To solve this problem, the Snow Leopard Trust and Microsoft agreed to partner with each other. Working with the Azure Machine Learning team, the Snow Leopard Trust built an image classification model that uses deep neural networks at scale on Spark [4]

\subsection{Protecting Honeybees}

As reported by Inverse, one third of the bee population died in US in 2016. Varroa mites possess a biggest threat to bees resulting in decline in bee population and directly impacting the global food supply. Due to the difficulty and time associated with monitoring these bees, Swedish beekeeper and inventor Björns Lagerman is turning to deep learning and smart phones to help preserve bee colonies. BeeScanning is a smart phone app that allows one to upload image of a bee colony upon which it uses an image recognition technique to make a distinction between infected and noninfected bees as infected bees could easily be identified with mites feeding on the back of these bees. BeeScanning will be providential to beekeepers to help protect existing colonies. It 
can also help identify mite resistant bees [5].

\subsection{Identifying cruel and good animal selfies using image classification}

The practice of using wild animals as props for tourists to post on social media has become a trend in recent days as a result of which these animals are suffering both in front of and behind the camera. The growing demand for harmful wildlife selfies is a serious animal welfare concern. Recent investigation in the Amazon revealed that over 20 percent of the species involved are threatened by extinction and over 60 percent are protected by international law. World Animal Protection a charity organization for animals partnered with Grassriots to develop an image recognition system that makes use of trained social listening algorithms and machine learning to differentiate good selfies with bad ones amongst thousands of photographs posted on social media like Instagram, Twitter Facebook etc. The model is trained to consider a selfie as "bad" if someone is holding, hugging or improperly interacting with the animal and a selfie as "good" if the photo was taken in animal's natural habitat without any interaction with the humans [6].

\section{CONCLUSION}

By aptly leveraging the power of machine learning and exploring the deepest corners, the above stated uses could prove to be pioneering and revolutionary in the field of AI. Also, one can observe that with the flexibility of the above stated algorithms, a model if trained to identify a particular animal or a species, it could easily be trained to identify others by providing it with sufficient training data to learn. Not only patterns in an image but also patterns in acoustic signals generated by animals could be decrypted to understand the animal behaviour.

\section{FUTURE SCOPE}

By using sophisticated image detection and video image classification techniques,, a greater advancement could be made in other areas as well . For example, images collected by drones or camera traps on tree tops could be used to collect endangered bird images that could help the ornithologists to study bird behaviour. If a sufficient investment is made towards using GPUs, acoustic sensors, camera traps and drones, it would be very easy to collect a huge amount of data to train these models with less human intervention.

\section{REFERENCES}

[1] Rekha Chahar, Priyanka Soni, "A Study of Image Processing in Agriculture for Detect the Plant Diseases",IJCSMC vol. 4 , July 2015.

[2] Mohammed Sadegh Norouzzadeh, Anh Nguyen, Margaret Kosmala, Ali Swanson, Craig Packer, Jeff Clune "Automatically identifying wild animals in camera trap images with deep learning".

[3] Frederic Mairea, Luis Mejiasa,b, Amanda Hodgsonc, Gwenael Duclos "Detection of Dugongs from Unmanned Aerial Vehicles"

[4] Snow Leopard Trust and Microsoft "Saving Snow Leopards with Deep Learning and Computer Vision on Spark".

[5] Björn Lagerman and team "BeeScanning" project.

[6] World Animal Protection and Grassriots "Wildlife Selfie Code"project. 